\title{
LEVANTAMENTO DE PLANTAS INVASORAS NO CAMPUS DA UFSM - SANTA MARIA, RS - MAGNOLIOPSIDA
}

\author{
Luciana Zago Ethur \\ Bolsista Aperfeiçoamento FAPERGS \\ Departamento de Biologia - UFSM - Santa Maria, RS \\ Sônia Maria Eisinger \\ Departamento de Biologia - Centro de Ciências Naturais e Exatas \\ UFSM - Santa Maria, RS \\ Mara Rejane Ritter \\ Departamento de Botânica - Instituto de Biociêcias \\ UFRGS - Porto Alegre, RS
}

RESUMO

O trabalho teve como objetivo o levantamento das plantas invasoras em lavouras experimentais no Campus da Universidade Federal de Santa Maria, RS. Foram totalizadas 170 espécies de Magnoliopsida distribuídas em 34 familias, sendo Asteraceae a família melhor representada com 60 espécies.

\section{SUMMARY}

The aim of this work was to classify weed plants present on experimental fields in the Campus of the Federal University of Santa Maria (UFSM), RS. It has been totalized 170 species from Magnoliopsida spread on 34 families, among them the Asteraceae family was the most expressive with 60 species. 


\section{INTRODUÇÃO}

O municipio de Santa Maria está localizado na região fisiográfica da Depressão Central do Rio Grande do Sul. Nesta Região predominam áreas de cultivo de campos utilizados principalmente na criação extensiva de bovinos. Encontram-se ainda, manchas de vegetação arbustiva, arbórea e matas ciliares.

O Campus da Universidade Federal de Santa Maria compreende uma área total de 1182 ha, sendo 210 ha ocupados com área construida, e o restante com áreas experimentais de campo, algumas manchas de Pinus e Eucalyptus, além de um arroio e açudes.

As espécies invasoras são também denominadas de plantas daninhas e compreendem vegetais que crescem em locais indesejados (LORENZI, 1991). Já para HAMEL \& DANSEREAU (1986), o termo erva daninha corresponde a um conceito popular e inclui, normalmente, qualquer planta que cause contrariedade ao homem. O conhecimento destas plantas no Brasil é muito restrito (ARANHA et alii, 1988).

As plantas daninhas desenvolveram grande habilidade à sobrevivência, atribuída a mecanismos, tais como grande agressividade competitiva, grande produção, facilidade de dispersão e grande longevidade das sementes (LORENZI, 1991). A identificação das espécies é fundamental para a pesquisa voltada ao controle das plantas daninhas.

O presente trabalho teve como objetivo o levantamento das espécies pertencentes à classe Magnoliopsida, invasoras de lavouras experimentais do Campus da Universidade Federal de Santa Maria.

\section{MATERIAL E MÉTODOS}

As coletas foram realizadas na área do Campus da Universidade Federal de Santa Maria (UFSM). Foram consideradas 04 (quatro) áreas de lavouras experimentais onde foram cultivados cereais, cana-de-açúcar e principalmente milho. As áreas de lavoura eram semelhantes entre si, com exceção da área 04, que possuia sistema de irrigação. O período de coleta estendeu-se de outubro de 1992 a fevereiro de 1995.

O solo das áreas experimentais é podzólico vermelho-amarelo, de acordo com o mapeamento correspondente à unidade Santa Maria.

A visitação às áreas era periódica, com exceção do período do inverno. Na primavera e verão as coletas foram quinzenais. Todas as espécies férteis, pertencentes à classe Magnoliopsida, foram coletadas e posteriormente secadas, catalogadas e identificadas. As exsicatas foram 
incorporadas ao herbário (SMDB) do Departamento de Biologia da UFSM.

No presente trabalho, seguiu-se o Sistema de Classificação de CRONQUIST (1981).

\section{RESULTADOS E DISCUSSÃO}

A área 01 foi a mais utilizada experimentalmente, ou seja, a que apresentou maior rotatividade de culturas durante o periodo de coleta. Este fato contribuiu para o elevado número de espécies presentes (Figura 1). No final de 1993 foi plantado Eucalyptus em parte desta área, o que prejudicou as coletas.

As demais áreas $(02,03$ e 04), possuiam culturas anuais ou bianuais e, por serem aradas eventualmente, geraram problemas nas coletas, o que refletiu no baixo número de espécies, principalmente na área 04 (Figura 1). Salienta-se ainda, o fato de que a área 03, durante certo período, não foi cultivada, e na área 02, foi aplicado herbicida na cultura de milho, em 1994.

Figura 1 - Número de espécies por área de coleta

\section{$\mathbf{N}^{0}$ de espécies}

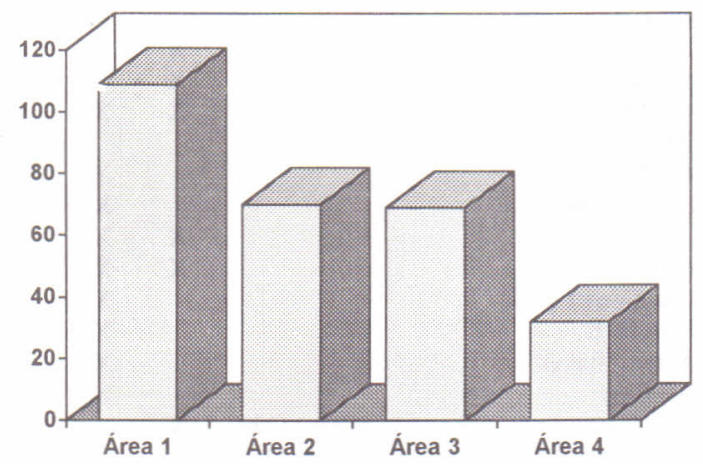

Áreas de Coleta

Foram identificadas 170 (cento e setenta) espécies, distribuidas em 34 (trinta e quatro) famílias de Magnoliopsida, conforme Tabela 1. As familias mais representadas foram Asteraceae (60 espécies), Fabaceae (14 espécies), Lamiaceae ( 8 espécies), Scrophulariaceae e Verbenaceae (7 espécies), respectivamente (Figura 2).

Segundo KISSMANN \& GROTH (1992) a familia Asteraceae é uma das mais expressivas, com um número de gêneros ainda não bem definidos. Como plantas infestantes, contudo, as compostas têm uma excepcional importância, pelo grande número de espécies e pela agressividade que algumas apresentam. 
TABELA 1 - Relação das famílias e espécies presentes nas áreas de estudo.

\begin{tabular}{l}
\hline \hline FAMÍLIAS / ESPÉCIES \\
\hline AIZOACEAE \\
Mollugo verticillata L. \\
AMARANTHACEAE \\
- Alternanthera brasiliana (L.) Ktze. \\
- Alternanthera philoxeroides (Mart.) Griseb. \\
- Amaranthus viridis L. \\
- Gomphrena celosioides Mart. \\
- Iresine diffusa H.\& B. ex Willd. \\
- Pfaffia tuberosa (Spreng.) Hicken.
\end{tabular}

\section{APIACEAE}

- Apium leptophyllum (Pers.) F. Muell.

- Centela asiatica (L.) Urban

- Eryngium elegans Cham. e Schl.

\begin{tabular}{ll} 
& ÁR \\
1 & 2 \\
\hline & $\mathrm{X}$ \\
\hline
\end{tabular}

ÁREAS

$3 \quad 4$

s

\section{ASCLEPIADACEAE}

- Oxypetalum arnotiamum Buek.

$\mathrm{X}$

$\mathrm{X}$

$\mathrm{X}$

$\mathrm{X} \quad \mathrm{X}$

$\mathrm{X}$

$\mathrm{X} \quad \mathrm{X}$

$\mathrm{X} \quad \mathrm{X}$

$\mathrm{X}$

$\begin{array}{llll}X & X & X & X \\ & X & & \\ X & & & X\end{array}$

ASTERACEAE

- Acanthospermum australe (Loefl.) Ktze.

- Achyrocline satureioides (Lam.) DC.

- Ageratum conyzoides L.

- Ambrosia elatior L.

- Artemisia verlotorum Lam.

- Aspilia montevidensis (Spreng.) Ktze.

- Aster squamatus (Spreng.) Hieron. var. squamatus

- Baccharis punctulata DC

- Baccharis spicata (Lam.) Baill.

- Bidens pilosa L.

- Bidens subalternans DC.

- Chaptalia integerrima (Vell.) Burk.

- Chaptalia runcinata H. B. K.

- Chaptalia simuata (Less.) Baker

- Conyza blakei (Cabr.) Cabr.

- Conyza bonariensis (L.) Cronquist var. angustifolia (Cabr.) Cabr.

- Conyza bonariensis (L.) Cronquist var. microcephala (Cabr.) Cabr.

- Conyza floribunda H. B. K.

- Eclipta prostata (L.) L.

- Elephantopus mollis H. B. K.

- Erechthites hieracifolia (L.) Rafin.

- Erechthites valerianifolia (Wolf.) DC.

- Eupatorium bupleurifolium DC.

- Eupatorium candolleamum Hook. et Arn.

- Eupatorium hecatanthum (DC.) Baker

- Eupatorium inulaefolium H. B. K.

- Eupatorium laevigatum Lam.

- Eupatorium macrocephalum Less.

- Eupatorium verbenaceum DC.

- Facelis retusa (Lam.) Sch. Bip.

- Galinsoga parviflora Cav.

- Gamochaeta americana (Mill.) Wedd.

- Gamochaeta falcata (Lam.) Cabr.

- Gamochaeta filaginea (DC.) Cabr. 
Tabela 1 - continuação

- Gamochaeta simplicicaulis (Willd.) Cabr

- Gamochaeta spicata (Lam.) Cabr.

- Gnaphalium gaudichaudiamum DC.

- Hypochoeris brasiliensis (Less.) Griseb.

- Hypochoeris pampasica Cabr.

- Jaegeria hirla (Lag.) Less.

- Mikania micrantha H. B. K.

- Orthopappus angustifolius (Sw.) Gleason

- Pluchea sagittalis (Lam.) Cabr.

- Porophyllum lanceolanum DC.

- Porophyllum ruderale (Jacq.) Cass.

- Pterocaulon alopecuroides (Lam.) DC.

- Pterocaulon polystachyum DC.

- Senecio brasiliensis (Spreng.) Less.

- Senecio heterotrichius DC.

- Senecio oxiphyllus DC.

- Senecio leptolobus DC.

- Senecio selloi (Spreng.) DC.

- Solidago chilensis Meyer

- Soliva macrocephala Cabr.

- Soliva pterosperma (Juss.) Less.

- Sonchus asper (L.) Hill.

- Sonchus oleraceus L.

- Taraxacum officinale Weber

- Vernonia flexuosa Sims.

- Vernonia hipochaeris DC.

\section{BORAGINACEAE}

- Heliotropium indicum L.

\section{BRASSICACEAE}

- Capsella bursa - pastoris (L.) Medik.

- Coronopus didymus (L.) Smith.

- Lepidium ruderale $\mathrm{L}$.

- Raphamis raphanistrum L.

CAMPanulaceae

- Triodanis biflora (R. et Pav.) Greene

- Wahlenbergia linarioides (Lam.) DC

\section{CARYOPHYLLACEAE}

- Cerastium rivulariastrum Möschl et Ped.

- Drymaria cordata (L.) Willd.

- Paronichia setigera (Gill. ex. Hook.) F. Heim

- Silene gallica L.

- Spergula arvensis $\mathrm{L}$.

- Spergula grandis Pers.

CISTACEAE

- Halimium brasiliensis (Lam.) Grosser.

CONVOLVULACEAE

- Ipomoea grandifolia (Dam.) O'Donell
$\mathrm{X}$

$\mathrm{X}$

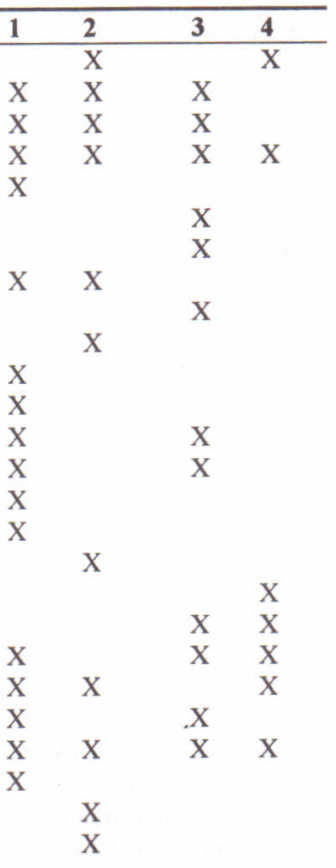

$\begin{array}{lll} & & \mathrm{X} \\ \mathrm{X} & \mathrm{X} & \\ & \mathrm{X} & \mathrm{X} \\ \mathrm{X} & \mathrm{X} & \\ & & \\ \mathrm{X} & \mathrm{X} & \\ \mathrm{X} & & \end{array}$

$\mathrm{X}$

$\begin{array}{llll} & & \mathrm{X} & \\ \mathrm{X} & \mathrm{X} & & \\ \mathrm{X} & \mathrm{X} & & \mathrm{X} \\ \mathrm{X} & & \mathrm{X} & \\ \mathrm{X} & \mathrm{X} & & \end{array}$

$\begin{array}{llll}\mathrm{X} & \mathrm{X} & \mathrm{X} & \mathrm{X}\end{array}$

EUPHORBIACEAE

- Phyllanthus stipulatus (Raf.) Webster 
FABACEAE

- Aeschynomene sp.

X

- Crotalaria pilosa Will

- Desmanthus tatuhyensis Hoehne

- Desmodium incanum DC.

- Desmodium barbatum (L.) Benth.

- Lathyrus sativus L.

- Lotus corniculatus L.

- Medicago polymorpha L.

- Melilotus indicus (L.) All.

- Stylosanthes montevidensis Vog.

- Trifolium vesiculosum Savi.

- Vicia angustifolia L.

- Vicia sativa L.

- Vicia villosa Roth

$\begin{array}{lll} & \mathrm{X} & \\ & \mathrm{X} & \\ \mathrm{X} & & \mathrm{X} \\ \mathrm{X} & & \\ \mathrm{X} & & \\ \mathrm{X} & & \\ \mathrm{X} & & \mathrm{X} \\ \mathrm{X} & \mathrm{X} & \\ \mathrm{X} & & \\ & \mathrm{X} & \end{array}$

\section{GESNERIACEAE}

- Corytholoma allagophyllum (Mart.) Fritsch.

\section{HYPERICACEAE}

- Hypericum brasiliense Choisy

\section{LAMIACEAE}

- Hyptis cf. alata (Raf.) Shin

- Hyptis brevipes Poit.

- Hyptis laphacea Benth

- Hyptis mutabilis (Rich) Briquet

- Peltodon longipes St. Hil.

- Scutellaria arvensis L.

- Scutellaria racemosa Pers

- Stachys arvernsis L.

\section{LYTHRACEAE}

- Cuphea carthagenensis (Jacq.) Macbr.

- Heimia salicifolia Link

$\begin{array}{llll} & & \mathrm{X} & \\ \mathrm{X} & & \mathrm{X} & \\ \mathrm{X} & \mathrm{X} & & \\ \mathrm{X} & & & \\ \mathrm{X} & & & \\ \mathrm{X} & \mathrm{X} & & \mathrm{X} \\ \mathrm{X} & & & \end{array}$

MALVACEAE

- Modiolastrum malvifolium (Griseb.) K. Sehum

- Sida rhombifolia L.

- Sida urens L.

$\begin{array}{llll}X & & & \\ X & X & X & \text { X } \\ X & & & \text { X }\end{array}$

MELASTOMATACEAE

- Tibouchina gracilis (Bonpl.) Cogn. X

\section{OLEACEAE}

-Menodora integrifolia (Cham. et Sch.) Steudel

$\mathrm{X}$

$$
\mathrm{X}
$$

\section{ONAGRACEAE}

- Ludwigia c f. bonariensis (Mich) Hara

- Ludwigia sericea (Camb.) Hara

- Ludwigia c f. suffruticosa (L.) Hara

- Oenothera indecora Camb.<smiles>[X]CC</smiles>

- Oxalis floribunda Lehman 
Tabela 1 - continuação

- Oxalis mallabolba Cav.

- Oxalis serpens St. Hil. var. serpens

2

34

X

PLANTAGINACEAE

- Plantago tomentosa Lam.

X X

X

POLYGALACEAE

- Monnina oblongifolia Arechav.

- Polygala brasiliensis L.

$\mathrm{X}$

$\mathrm{X}$

\section{POLYGONACEAE}

- Muehlembeckia sagittifolia Meiss

- Polygomim hydropiperoides Mich.

- Polygomum persicarioides H. B. K.

- Polygomum punctatum Elliot

- Polygomum setaceum Bald.

- Rumex obovatus Danser

$\mathrm{X}$

$\begin{array}{lll} & & \mathrm{X} \\ \mathrm{X} & & \\ \mathrm{X} & & \mathrm{X} \\ \mathrm{X} & & \\ \mathrm{X} & \mathrm{X} & \end{array}$

PORTULACACEAE

- Portulaca oleracea L.

- Portulaca papulifera Legr.

- Portulaca striata Poellnitz

- Talimum patens (Jacq.) Willd.

$\mathrm{X}$

\section{PRIMULACEAE}

- Anagallis arvensis L.

$\begin{array}{ll}X & \\ X & \\ X & \\ X & X\end{array}$

\section{RUBIACEAE}

- Borreria verticillata (L.) G. F. W. Meyer

- Diodia brasiliensis Spreng.

- Relbunium hypocarpium (L.) Hemsley

- Richardia brasiliensis Gomez

X

\section{SCROPHULARIACEAE}

- Castilleja communis Benth.

- Gerardia communis Cham. et Sch.

- Linaria texaria Scheele

- Mecardonia tenella (Cham.) Pennell

- Scoparia dulcis L.

- Scoparia montevidensis (Spreng.) Fries

- Veronica peregrina L.

$\mathrm{X}$

X X

\section{SOLANACEAE}

- Solanum americamum Mill.

- Solamum chenopodioides Lam.

- Solamum fastigiatum Willd.

- Solanum hieronymi O. Ktze.

- Solanum paniculatum L.

- Solanum sisymbriifolium Lam.

$\begin{array}{llll}\mathrm{X} & \mathrm{X} & & \\ & \mathrm{X} & & \\ \mathrm{X} & \mathrm{X} & & \\ & & & \\ & & & \\ \mathrm{X} & & & \\ & & \mathrm{X} & \\ \mathrm{X} & \mathrm{X} & \mathrm{X} & \\ \mathrm{X} & & \mathrm{X} & \mathrm{X} \\ \mathrm{X} & \mathrm{X} & \mathrm{X} & \mathrm{X} \\ & & \mathrm{X} & \\ \mathrm{X} & & & \end{array}$

$\mathrm{X}$

TURNERACEAE

- Piriqueta selloi Urban

- Turnera sp.

$\begin{array}{llll}\mathrm{X} & \mathrm{X} & \mathrm{X} & \mathrm{X} \\ \mathrm{X} & \mathrm{X} & & \\ \mathrm{X} & \mathrm{X} & & \\ & & & \mathrm{X} \\ \mathrm{X} & & \mathrm{X} & \\ \mathrm{X} & \mathrm{X} & & \mathrm{X}\end{array}$

URTICACEAE

- Parietaria debilis Forst. 
Tabela 1 - continuação

\begin{tabular}{llll}
1 & 2 & 3 & 4 \\
\hline
\end{tabular}

VERBENACEAE

- Glandularia megapotamica (Spreng.) Cabr.

- Glandularia peruviana (L.) Small

- Lantana camara L.

- Verbena bonariensis $\mathrm{L}$.

- Verbena litoralis H. B. K.

- Verbena montevidensis Spreng.

- Verbena rigida Spreng.

\begin{tabular}{llll}
$\mathrm{X}$ & & & \\
$\mathrm{X}$ & $\mathrm{X}$ & & \\
$\mathrm{X}$ & $\mathrm{X}$ & $\mathrm{X}$ & $\mathrm{X}$ \\
$\mathrm{X}$ & $\mathrm{X}$ & $\mathrm{X}$ & \\
$\mathrm{X}$ & & $\mathrm{X}$ & \\
$\mathrm{X}$ & $\mathrm{X}$ & & $\mathrm{X}$ \\
& & & \\
$\mathrm{X}$ & $\mathrm{X}$ & & \\
$\mathrm{X}$ & & $\mathrm{X}$ & \\
\hline
\end{tabular}

VIOLACEAE

- Hybanthus serratus (Phil.) Harsler - Ionidium glutinosum Malme

FIGURA 2 - Número de espécies por familia nas áreas de coleta

$\mathrm{N}^{\circ}$ de espécies

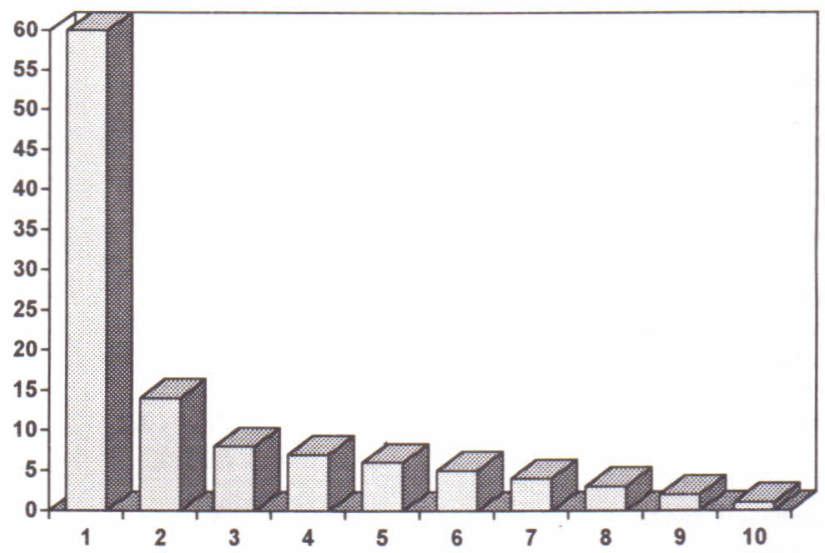

\section{LEGENDA:}

Famílias

1 - Asteraceae

2 - Fabaceae

3 - Lamiaceae

4 - Scrophulariaceae e Verbenaceae

5 - Amaranthaceae, Caryophyllaceae e Polygonaceae

6 - Oxalidaceae e Solanaceae

7 - Brassicaceae, Onagraceae, Portulacaceae e Rubiaceae

8 - Malvaceae e Apiaceae

9 - Campanulaceae, Lythraceae, Polygalaceae, Turneraceae e Violaceae 10 - Outras

Os gêneros com maior riqueza de espécies foram: Eupatorium (7 espécies), Solamum (6 espécies), Gamochaeta (5 espécies) e Senecio (5 espécies). 
Algumas espécies de Asteraceae foram encontradas em todas as áreas, de accordo com a Tabela 1, como é o caso da serralha (Sonchus oleraceus L.), chicória-do-campo (Hypochoeris brasiliensis (Less.) Griseb.) e picão (Bidens pilosa L.). Este, no Brasil, segundo KISSMANN \& GROTH (1992) e LORENZI (1991) está presente em quase todo o território nacional, sendo uma das mais sérias infestantes de lavouras, e que hoje já está sendo considerada "planta daninha" em aproximadamente 40 países. Espécies das demais familias encontradas em todas as áreas foram: Sida rhombifolia L., Ipomaea grandifolia (Dam.) O'Donell, Verbena bonariensis L., Apium leptophyllum (Pers.) Muell., Solanum americamum Mill. e Scoparia dulcis L. (Tabela 1).

\section{BIBLIOGRAFIA CITADA}

ARANHA, C; LEITÃo FILHO, H de; YAHN, C. A Sistemática de Plantas Invasoras. Campinas : Instituto Campineiro de Ensino Agricola, 1988. 174 p.

CRONQUIST, A. An integrated system of classification of flowering plants. New York Columbia University Press, 1981. 1262 p.

HAMEL, A.; DANSEREAU, P. O aspecto ecológico do problema das ervas daninhas. Roessléria, Porto Alegre, 8 (2): 132-178, 1986.

KISSMANN, K. G.; GROTH, D. Plantas infestantes e nocivas. São Paulo : BASF, 1991-1992. tomo II. $798 \mathrm{p}$.

LORENZI, H. Plantas daninhas do Brasil. 2. ed. Nova Odessa : Plantarum, 1991. 440 p. 
